\title{
Surgical Audit: Do Numbers Provide Good Training?
}

\author{
Lt Col ID Crate \\ FRCS(Ed), RAMC* \\ Lt Col CL Griffiths \\ $M B, F R C S(E n g)$, RAMC
}

Queen Elizabeth Military Hospital, Stadium Road, Woolwich, London SE18 4QH SUMMARY: There has been a growing realisation that good resource planning requires effective measurement of $\stackrel{\square}{ }$
surgical workload by good surgical audit. This paper examines the general surgical workload at the Army'sco
tertiary referral centre, utilising the ratio of operative workload expressed as Intermediate Equivalents to total $\overline{ }$
Service Equivalent Value of a Surgical team. A suggested format to enable inclusion of specialist non-surgical-
therapies is proposed. The limitations of "number crunching" when assessing the value of higher surgical trainingc్
are discussed.

\section{Introduction}

With the advent of the health service reforms, Trust Hospitals have increasingly demonstrated that provision of good health care requires accurate audit of present workload, so that future workload can be predicted and budgeted for. Surgical audit can now be used both as an internal system of quality control and as an indicator of present and future resource requirements, comparison of previous workload reflect trends in referral and workload patterns. The principal model for assessment of surgical workload on a quantitative basis has been the work of Collins $(1,2)$ defining the value of the Intermediate Equivalent (IE) in assessing operative workload by complexity of procedure and the Service Equivalent Value (SEV) as a measure of effective contribution of surgical trainees to patient care.

Recent litigation has brought to the fore questions about the appropriateness of higher professional training, especially in light of European Commission directives on this matter. Recent communications from the Chief Medical Officer (3) have highlighted the concerns of the European Commission, and the proposed responses from the United Kingdom. The question of what trainers, trainees and society want from surgical training has yet to be fully addressed, as have the problems of quality and quantity in surgical training. Guideline training programs have been devised to fit in with current training practice(4), but this may have to be altered to fit in with newer training programs.

The Queen Elizabeth Military Hospital is the tertiary referral centre for the Army, seeing referrals from throughout the world, and a proportion of the local civilian population. The Oncology unit is actually staffed by surgeons, but sees and treats both surgical and medical oncology cases taking supra-specialist advice where necessary. This paper examines the numerical aspects of case load at this unit, and begs the question as to whether numbers alone provide surgical training, or whether the? quality of caseload can be taken into account using the usual surgical audit parameters.

\section{Method}

The surgical caseload of the oncology consultant firos? was examined from 1st December 1992 to 28th Febru产y 1993. The oncology unit has its own audit packa\&e utilising DBase 4 running on a 286 processor which ho demographic and procedure details for all patients sire July 1992. A data query requiring all operative proceduke during the 3 month period ending 28th February 1993 used to extract relevant data. Chemotherapy episodes a d treatments are also held on the same database in the sa: format, and can be retrieved in the same manner. Theg Private Patients Plan "Introductory Notes on the Scheduled of Surgical Operations and Procedures" (5) was used to assign the degree of complexity of the procedures, this윽 was translated to a numerical value as recommended by Collins (2), shown in Table 1.

Table 1

\begin{tabular}{lll}
\hline \multicolumn{1}{c}{$\begin{array}{c}\text { Operative Workload } \\
\text { Intermediate Equivalents }\end{array}$} & \\
\hline Minor & $=0.5$ \\
Intermediate & $=1.0$ \\
Major & $=1.75$ \\
Major + & $=$ & 2.2 \\
Complex Major & $=4.0$ \\
\hline
\end{tabular}

(After Collins, Annals, RCSEng 1991) 
The total Service Equivalent Value of the Oncology team was calculated according to Collins (2), as shown in Table 2.

Table 2

\section{Service Equivalent Values (SEV)}

\begin{tabular}{lcc}
\hline Surgical Team & SEV & Contribution to Team \\
1 Consultant & 1.0 & 1.0 \\
$1 / 2$ Registrar & 0.75 & 0.37 \\
1 SHO & 0.5 & 0.5 \\
Total SEV of Team $=1.87$ &
\end{tabular}

(After Collins, Annals, RCSEng 1991)

Assigning a quantitative measure to chemotherapy episodes was achieved by estimating the total time required of senior medical staff per patient during a course of chemotherapy, and dividing this by the number of inpatient admissions during the course of chemotherapy per patient. Very few patients had chemotherapy as outpatients, most requiring an overnight stay due to the wide geographical distribution of our patient base. Time and resource estimation took account of clinical examination, ward investigation, preparation for specialist (laboratory and radiographic) investigation, ward procedures directly related to chemotherapy administration and on-ward counselling by medical staff of Consultant or Senior Registrar status. Time spent in other departments for investigation was not included in this assessment. Nursing procedures and counselling were excluded from this audit. A numerical value (an intermediate equivalent) was assigned on the basis of one IE per hour of clinical time. Each admission for chemotherapy was documented in the notes including history and examination findings. Each patient had a full clinical examination prior to each cycle of chemotherapy, documenting new symptoms or side effects of therapy. Investigations for each admission varied depending on the protocol for each chemotherapy regimen. We found that each admission, with history, examination and counselling, took a minimum of one hour. Only half of this time actually related to senior medical staff and therefore we assigned an IE value of 0.5 to each chemotherapy admission.

This permits the calculation of a ratio of Intermediate Equivalents per Service Equivalent Value (IE/SEV) for the team, over the period of study. It is not unreasonable to expect a consistent workload over a year, and this IE/SEV ratio can be multiplied by a factor of 4 to give an approximate IE/SEV ratio for the year. This was compared with the expected IE/SEV ratio for a one Consultant team working in a District General Hospital using the data from Collins(1) and applying the same calculations.

During the time studied and for the succeeding three months, this firm was limited to two elective operating sessions per week due to theatre staffing constraints.

\section{Results}

A total of 78 patients had 98 surgical procedures performed (Table 3 ). Where more than one procedure wa⿳亠㐅冋 performed, both procedures were assigned a value. Bot $\hat{\beta}$ G019 and G091 were thoraco-abdominal procedures Procedure complexity value varied from 0.5 to 4.0 . D total of 46 chemotherapy episodes were recorded for the duration of the study.

The IE/SEV ratio for the study period, including chemotherapeutic episodes, was 60.56 , giving an IE/SE ratio of 242.22 for the year, but 193.04 for purel operative work.

\section{Discussion}

The IE/SEV ratio for a consultant team working in 을 District General Hospital, based upon suggested norms b $\vec{\omega}$ Collins(1) would be 508 calculated from 900 intermediats equivalents per year with a Consultant, half shar@ Registrar, half share Senior House Officer and 2 Clinic: Assistant sessions per week, giving a SEV of 1.77. This figure depends upon a theatre availability of found operating sessions per week; if this is limited to sessions, as in this unit, then the IE/SEV ratio for the yei\& would be 254 as compared with this unit's ratio of 193 fo purely operative work. It is important to remember few DGH surgical firms would undertake the mबire specialised work associated with an Oncology unit.解 first glance this suggests that operative training in $\mathbb{P}_{\mathrm{O}} i \mathrm{~s}_{\text {. }}$ tertiary referral centre is less valuable than that is $\mathbb{E}$ District General Hospital, and any surgeon in trainoำ enjoys the operating more than any other facet of train that is after all what most surgeons enjoy! The difficosit arises when one tries to quantify the non-operative side $\frac{}{6}$ the workload in a tertiary referral centre, which is a majő part of the daily workload in specialist centres. This un⿷ in any one month can expect to administer a minimum of 15 chemotherapy episodes, with all the attendant clinic $\mathbb{B}$ and investigative work that goes with it. More than thi: due to the unusual demography of our patient base, the unit sees far more of the unusual tumours affecting the young, further complicating assessment of workload. The medical management of oncological disease an complications of treatment are both complex an interesting, but impossible to quantify with currere surgical audit parameters. This facet of practice cag provide great benefits to the surgeon in training, honing. his medical management skills, and stimulating interest if subjects not normally the remit of the general surgeon in training. We feel our attempt to remedy this defect io current surgical audit parameters is beneficial in definin? the training potential of more specialised units. Tho question has to be asked: how good a parameter of the adequacy of surgical training is provided purely by auds of operative procedures? A wide ranging comparison og operative workload in tertiary referral centres compare స్ట with District General Hospitals is unlikely to answer thio question; our opinion is that a wide range of IE/SE 
Table 3

Operation Code and Complexity Value

\begin{tabular}{|c|c|c|c|c|c|c|c|}
\hline OPCS CODE & VALUE & OPCS CODE & VALUE & OPCS CODE & VALUE & OPCS CODE & VALUE \\
\hline A651 & 1.0 & B274 & 1.75 & M135 & 0.5 & M451 & 1.0 \\
\hline B274 & 1.75 & B274 & 1.75 & $\mathrm{~N} 101$ & 1.0 & $\mathrm{~N} 171$ & 0.5 \\
\hline B283 & 1.0 & B283 & 1.1 & $\mathrm{~N} 171$ & 0.5 & $\mathrm{~N} 171$ & 0.5 \\
\hline B283 & 1.0 & B363 & 0.5 & $\mathrm{~N} 171$ & 0.5 & $\mathrm{~N} 303$ & 1.0 \\
\hline G019 & 4.0 & G091 & 4.0 & S059 & 0.5 & S065 & 0.5 \\
\hline G33 & 1.75 & L91 & 0.5 & S37 & 0.5 & S065 & 0.5 \\
\hline G451 & 0.5 & G451 & 0.5 & S068 & 0.5 & S 365 & 0.5 \\
\hline G451 & 0.5 & G451 & 0.5 & T104 & 1.75 & T104 & 1.75 \\
\hline G451 & 0.5 & $\mathrm{H} 22$ & 1.0 & $\mathrm{~T} 12$ & 0.5 & T203 & 1.0 \\
\hline $\mathrm{H} 22$ & 1.0 & $\mathrm{H} 22$ & 1.0 & T203 & 1.0 & T203 & 1.0 \\
\hline $\mathrm{H} 221$ & 1.0 & $\mathrm{H} 221$ & 1.0 & T208 & 1.0 & T209 & 1.75 \\
\hline $\mathrm{H} 28$ & 0.5 & $\mathrm{H} 412$ & 1.0 & T209 & 1.75 & $\mathrm{~T} 223$ & 1.0 \\
\hline $\mathrm{H} 443$ & 0.5 & H511 & 0.5 & $\mathrm{~T} 258$ & 1.0 & $\mathrm{~T} 351$ & 1.75 \\
\hline H443 & 0.5 & H491 & 0.5 & $\mathrm{~T} 351$ & 1.75 & T351 & 1.75 \\
\hline $\mathrm{H} 443$ & 0.5 & $\mathrm{H} 281$ & 0.5 & T43 & 1.0 & T43 & 1.0 \\
\hline H443 & 0.5 & $\mathrm{H} 281$ & 0.5 & T591 & 1.0 & T872 & 1.0 \\
\hline $\mathrm{H} 443$ & 0.5 & $\mathrm{H} 281$ & 0.5 & T872 & 1.0 & W364 & 0.5 \\
\hline $\mathrm{H} 443$ & 0.5 & E249 & 0.5 & W364 & 0.5 & W364 & 0.5 \\
\hline $\mathrm{H} 443$ & 0.5 & $\mathrm{H} 524$ & 0.5 & W364 & 0.5 & & \\
\hline
\end{tabular}

H562

J088

1.0

H604

1.0

J088

J088

2.2

$2.2 \quad$ J088

2.2

TOTAL

SEV

IE/SEV

J088

L851

1.0

0.5

L874

0.5

L851

1.0

L852

1.0

IE/SEV PER YEAR

L874

0.5

L853

1.0

CHEMOTHERAPY EPISODES

L91

0.5

M451

IE/SEV (with chemotherapy)

IE/SEV PER YEAR (w.c.)

ratios would be produced, which would not reflect the true training value of the posts reviewed. Training is a subtle blend of service commitment and teaching, and the patient base is only one factor in this. The best measure of training is the progress of the trainee, and this is a very difficult parameter to quantify. The current system of annual review of a trainee by his or her peers is very subjective and relies upon personalities as well as data, but is probably the best that can at present be defended. Although it is true that operative experience by and large is greatest in the District General Hospitals, it must also be true that working with a committed team in a specialist referral centre provides life and work experience invaluable to the trainee, which nonetheless cannot be quantified.

\section{Conclusion}

We contend that, although operative exposure of the surgical trainee may be greater in a District General Hospital, good training requires that which cannot be easily quantified. Human and medical management skills are difficult to teach and even more difficult to learn, but are as essential a part of training as operative skills. Technical aspects of specialist treatment do not as yet have systems of quantification which allow objectivg assessment of their value in training. We present $\frac{8}{8}$ suggested format to remedy this defect in surgical audi? The present system of annual peer review, with all i $\overrightarrow{\vec{\sigma}}$ limitations, is the best system at present available for assessing the progress of the surgical trainee.

\section{REFERENCES}

1. Collins CD. The Work Programme and Workloa for a Consultant General Surgeon. Ann R Coll Surg Engl 1991 suppl: 73(2): 27-29.

2. Coll.INS CD. Recommended Values for use if Surgical Audit and Surgical Workload Analysis. Angु $R$ Coll Surg Eng/ 1991 suppl; 73(5): 94.

3. Chief Medical Officer. Working Group on Specialis medical Training. April 1993; PL/CMO(93)3.

4. Coluins CD. Guideline Training Program for Higheo Surgical Trainees in General Surgery Working in District General Hospital. Ann R Coll Surg Engl 199 宏 suppl; 72(3): 34-35.

5. Private Patients Plan: Introductory Notes on the Schedule of Surgical Operations and Proceduren Private Patients Plan, Eynsham House, Tunbridgo Wells. 